\section{Influência da renda sobre as despesas com alimentação fora do domicílio, no Brasil, 2002-2003}

\author{
Influence of income on food expenditures \\ away from home among Brazilian \\ families, 2002-2003
}

\author{
1 Núcleo de Pesquisas \\ Epidemiológicas em Nutrição \\ e Saúde, Universidade de \\ São Paulo, São Paulo, Brasil. \\ 2 Faculdade de Medicina, \\ Universidade de São Paulo, \\ São Paulo, Brasil. \\ 3 Faculdade de Saúde \\ Pública, Universidade de São \\ Paulo, São Paulo, Brasil. \\ Correspondência \\ R. M. Claro \\ Núcleo de Pesquisas \\ Epidemiológicas em Nutrição \\ e Saúde, Universidade de \\ São Paulo. \\ Av. Dr. Arnaldo 715, \\ São Paulo SP \\ 01246-904, Brasil. \\ rclaro@usp.br
}

\begin{abstract}
This study describes and evaluates the influence of income on the percentage of food expenditures away from home for Brazilian families. Food acquisition data from the National Household Budget Survey conducted from 2002 to 2003 (POF 2002/2003) by the Brazilian Institute of Geography and Statistics (IBGE) or National Census Bureau was used in the analysis. Information on food-and-drink expenditures away from home was analyzed. The influence of income on the share of food purchased away from home in the household budget, adjusted for socio-demographic variables, was analyzed through elasticity coefficients estimated in multiple linear regression. Food purchased away from home accounted for $21 \%$ of total food expenditures by Brazilian households. A 10\% increase in income increased the share of food purchased away from home by 3\%. Income elasticity was high, especially for the lowest income families. The results demonstrate an important influence of income on food expenditures away from home, and higher income is associated with a greater share of food purchased away from home.
\end{abstract}

Income; Nutrition Surveys; Food Consumption; Feeding
Rafael Moreira Claro 1

Renata Bertazzi Levy 1,2

Daniel Henrique Bandoni ${ }^{3}$

\section{Introdução}

O padrão de consumo alimentar no Brasil sofreu intensa e rápida modificação nas últimas décadas. A tendência observada nas regiões metropolitanas evidencia aumento no consumo de carnes, leite e derivados e óleos vegetais, além de substancial redução no consumo de cereais, leguminosas e tubérculos 1,2. Essas mudanças são complexas e envolvem diferentes aspectos, como: mudanças tecnológicas, novas formas de distribuição e marketing dos alimentos, variações na renda das famílias, conhecimentos dos indivíduos sobre alimentação, entre vários outros fatores 3,4 . Dentro deste cenário, algumas características do comportamento alimentar, entre elas o número de refeições e a alimentação fora do domicílio, são identificadas como determinantes centrais da modificação dos padrões alimentares 5. Segundo dados do U.S. Department of Agriculture (USDA), as refeições realizadas fora do domicílio apresentam maiores quantidades de gorduras totais, gorduras saturadas e colesterol, quando comparadas com a alimentação preparada no lar 6 .

Mesmo entre os países desenvolvidos, onde o consumo de alimentos fora de casa desempenha um papel de destaque na alimentação, poucos estudos se voltam à caracterização dessa importante parcela do consumo alimentar. Nos Estados Unidos, a participação do gasto com alimentação fora de casa aumentou nas últimas décadas, 
passando de menos de $20 \%$ em 1970 para $38 \%$ do gasto total com alimentação na década de 907 . No que se refere ao total de calorias da dieta, a participação da alimentação fora do lar saltou de 18\% em 1977 para $32 \%$ em 1995 8. Verifica-se que tanto em países desenvolvidos, quanto naqueles em desenvolvimento, a parcela da alimentação feita fora do domicílio tende a aumentar com a elevação do nível de renda das famílias, sendo o efeito do crescimento do poder aquisitivo especialmente importante entre os indivíduos com menor nível de renda 9,10.

No Brasil, dados da Pesquisas de Orçamentos Familiares (POF), do Instituto Brasileiro de Geografia e Estatística (IBGE), nas áreas metropolitanas do país, demonstraram que, entre 1988 e 1996, a participação dos gastos com alimentação fora do domicílio nos gastos totais com alimentação cresceu (de 24,4\% para 26,1\%) nas áreas metropolitanas do Centro-Sul, ao passo que, nas regiões Norte e Nordeste, verificou-se redução (de 25,6\% para 21,7\%) 11 .

Apesar da grande importância do consumo de alimentos fora do domicílio sobre o padrão de alimentação dos indivíduos, poucos estudos buscaram caracterizar essa forma de alimentação ou, ainda, quantificar possíveis fatores que a influenciem; na verdade, a maioria foi realizada em países desenvolvidos e sem representatividade nacional. Dessa maneira, o presente estudo tem como objetivo descrever e avaliar a influência da renda sobre a participação da alimentação fora do domicílio, no Brasil, por meio dos dados da POF realizada entre os anos de 2002/2003 12.

\section{Metodologia}

\section{Amostragem}

Dados coletados pela POF realizada pelo IBGE entre julho de 2002 e junho de 2003, em uma amostra probabilística de 48.470 domicílios brasileiros, serviram de base para este estudo 12. A POF 2002/2003 utilizou plano amostral complexo, por conglomerados, com sorteio dos setores censitários no primeiro estágio e de domicílios no segundo. Para o sorteio dos setores, a pesquisa procedeu, previamente, ao agrupamento dos 215.790 setores censitários do país existentes no Censo Demográfico de 2000 (http://www.ibge. gov.br), visando a obter estratos de residências com homogeneidade geográfica e sócio-econômica. Nesse agrupamento, consideraram-se a localização dos setores (região, unidade da federação, capital ou interior, área urbana ou rural) e o espectro de variação do nível sócio-econômico das famílias residentes (indicado pela média de anos de escolaridade dos chefes de domicílio, obtida na contagem populacional de 1996), de forma a constituir 443 estratos de domicílios. O número de setores sorteados em cada estrato foi proporcional ao número total de residências particulares permanentes no estrato. Por fim, sortearam-se aquelas, em cada setor, por amostragem aleatória simples, sem reposição. As entrevistas aos domicílios sorteados foram distribuídas uniformemente ao longo dos quatro trimestres de duração do estudo, a fim de reproduzir, em cada estrato, a variação sazonal de rendimentos e aquisições de alimentos.

A principal informação da POF 2002/2003 analisada neste estudo compreende os registros de aquisições de alimentos e bebidas para consumo fora de casa, anotados em uma caderneta pelos próprios moradores dos domicílios estudados (ou entrevistador, quando necessário) durante sete dias consecutivos. O curto período de referência empregado pela POF para o registro das despesas com alimentação não permite que se conheça o padrão usual de aquisição de alimentos de cada um dos lares estudados. Neste trabalho, optamos por utilizar como unidade de estudo agregados de domicílios que correspondem àqueles estudados em cada um dos 443 estratos amostrais da pesquisa, conferindo à análise um caráter ecológico. Assim, garantimos unidades de estudo com grande amplitude de variação geográfica e sócio-econômica, cujo padrão de aquisição anual de alimentos pode ser conhecido com grande precisão.

\section{Análise de dados}

\section{- Criação e definição das variáveis do estudo}

As despesas de cada morador da residência com alimentação fora de casa, registradas durante uma semana, foram somadas para determinar o total gasto em cada domicílio. Somaram-se a este gasto todas as despesas feitas com alimentos e bebidas (considerados como gastos com alimentos dentro do domicílio), para obtenção do gasto total com alimentação do domicílio. Com base nesse dado, determinou-se a participação da alimentação fora do lar sobre o total de gastos com alimentação. Adicionalmente, a quantidade total adquirida de cada alimento ou bebida para consumo em casa foi convertida em energia (kcal), empregando-se para tanto o aplicativo AQUINUT versão 1.0 (Núcleo de Pesquisas Epidemiológicas em Nutrição e Saúde, Faculdade de Saúde Pública, Universidade de São Paulo São Paulo, Brasil. http://hygeia.fsp.usp.br/nupens/ patual.htm), e dividida pelo número de dias da semana (a fim de se obterem valores diários). De- 
pois de convertidos para energia, os registros de um mesmo estrato de domicílios foram somados e divididos pelo total de moradores do estrato.

A renda média no estrato, expressa em $\mathrm{R} \$ /$ pessoa/mês, foi obtida dividindo-se a somatória do total de rendimentos mensais de todos os domicílios que integram o estrato pelo total de moradores neste; procedimento semelhante também foi adotado para os gastos com alimentação. De modo análogo, foram calculadas a média de idade dos moradores e a média de anos de escolaridade dos moradores com $18 \mathrm{ou}$ mais anos de idade no estrato. Foram também calculadas a proporção de mulheres e de chefes de família do sexo feminino, além da proporção de indivíduos com idade inferior a cinco anos e superior a 64 anos, para cada estrato. A macro-região geográfica onde este se localizava, agrupada em duas categorias: Norte-Nordeste e Centro-Sul (Centro-Oeste, Sul e Sudeste), e sua situação (capital, região metropolitana, outras áreas urbanas e área rural) complementaram a caracterização das unidades de estudo.

\section{- Procedimentos analíticos}

Inicialmente, investigou-se a associação entre a participação dos gastos com alimentação fora do domicílio (variável dependente) e características sócio-demográficas e econômicas dos estratos (variáveis explanatórias) por meio de modelos de regressão linear bivariados.

A associação entre a participação da alimentação fora do domicílio nos gastos com alimentação (variável dependente) e a renda domiciliar per capita (variável explanatória), ajustada para as variáveis de confusão (variáveis sócio-demográficas), foi estudada por meio de modelos de regressão linear múltiplos, utilizados para estimação de coeficientes de elasticidade. Este coeficiente indica o percentual de variação (positivo ou negativo) na participação da alimentação fora do domicílio frente a $1 \%$ de variação na renda (elasticidade-renda), ajustado pelas variáveis de confusão. O coeficiente de elasticidade corresponde ao coeficiente de regressão $(\beta)$ da variável explanatória em modelo de regressão múltipla do tipo log-log, no qual tanto a variável dependente (participação da alimentação fora do domicílio) quanto à independente (renda) sofreram transformação logarítmica 13,14. O modelo geral utilizado em nosso estudo pode ser definido como:

$\operatorname{Ln}(\%$ GAfd $)=\alpha+\beta_{1} \operatorname{Ln}(\mathrm{R})+\chi_{1}(\mathrm{VC})$ sendo \%GAfd a participação da alimentação fora do domicílio nos gastos totais com alimentação; $\mathrm{R}$ a renda mensal per capita (R\$) e VC variáveis de controle.
Para o modelo de regressão linear e de elasticidade, foram incluídos como variáveis de confundimento para a associação entre renda e alimentação fora de casa: macro-região geográfica e situação do estrato (região metropolitana, demais áreas urbanas ou área rural); média da idade dos moradores e dos anos de escolaridade dos moradores adultos; proporção de chefes de domicílio do sexo feminino, de mulheres, de crianças com menos de 5 anos e de adultos com 65 ou mais anos de idade. Para o modelo final foram incluídas as variáveis que alteraram em pelo menos 5\% a magnitude do coeficiente de regressão da participação dos gastos com alimentação fora do lar sobre a renda.

Todos os procedimentos analíticos deste estudo foram executados com o aplicativo Stata versão 9.2 (Stata Corp., College Station, Estados Unidos) e levaram em conta o delineamento complexo da amostra da POF 2002/2003 e o seu efeito sobre os erros-padrão de todas as estimativas apresentadas.

\section{Resultados}

A Tabela 1 apresenta a participação média do gasto com alimentação fora do domicílio nos gastos totais com alimentação, segundo variáveis sóciodemográficas e econômicas. A alimentação fora do lar respondeu por cerca de $21 \%$ do total dos gastos com alimentação no país. Essa participação aumentou significativamente conforme a elevação da renda e a maior proximidade à capital do estado. Conforme esperado, a aquisição de calorias para consumo no domicílio apresentou associação inversa com a parcela dos gastos com alimentação feitos fora de casa.

A Tabela 2 apresenta coeficiente de elasticidade-renda e coeficientes de regressão para as variáveis de confusão relativos à participação da alimentação fora do domicílio nos gastos totais com alimentação. A renda exerceu importante papel nos gastos com alimentação fora da residência, mesmo após controle das variáveis de confundimento. Desse modo, um incremento de $10 \%$ na renda aumentaria em cerca de $3 \%$ a participação da alimentação fora do domicílio nos gastos totais com alimentação. Nas capitais e áreas metropolitanas, também há uma maior participação dos gastos com alimentação fora do lar, assim como nos estratos com maior participação de mulheres como chefe da casa.

A Figura 1 descreve a variação da elasticidade-renda para a parcela dos gastos com alimentação feita fora do domicílio ao longo do espectro de variação da renda, estimado a com base em um modelo de regressão polinomial (descrito 
Valor médio da parcela dos gastos com alimentação fora do domicílio, segundo variáveis sócio-demográficas e econômicas. Brasil, 2002/2003.

\begin{tabular}{|c|c|c|}
\hline Variáveis & $\begin{array}{c}\text { Valor médio da parcela } \\
\text { dos gastos com alimentação } \\
\text { fora do domicílio (R\$) }\end{array}$ & Valor de $p$ * \\
\hline Renda domiciliar per capita $(\mathrm{R} \$)$ ** & & $<0,01$ \\
\hline 1 ㅇ $(67,83-298,57)$ & 15,93 & \\
\hline 2o $(300,94-493,27)$ & 20,07 & \\
\hline 3으 $(493,81-4.562,16)$ & 28,18 & \\
\hline Situação do domicílio *** & & $<0,01$ \\
\hline Capital & 28,11 & \\
\hline Região metropolitana & 22,61 & \\
\hline Outras áreas urbanas & 20,44 & \\
\hline Rural & 12,45 & \\
\hline 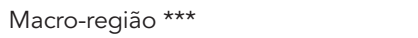 & & $<0,01$ \\
\hline Centro-Sul & 23,07 & \\
\hline Norte-Nordeste & 17,41 & \\
\hline Proporção do chefe de domicilio do & & $<0,01$ \\
\hline \multicolumn{3}{|l|}{ sexo feminino $(\%) * \star$} \\
\hline 1 으 $(0,00-23,20)$ & 17,11 & \\
\hline 2으 $(23,23-28,53)$ & 20,62 & \\
\hline 3으 $(28,59-75,28)$ & 26,15 & \\
\hline Proporção de indivíduos maiores & & 0,64 \\
\hline \multicolumn{3}{|l|}{ de 64 anos (\%) ** } \\
\hline 1 으 $(0,00-4,84)$ & 22,11 & \\
\hline 2으 $(4,88-7,07)$ & 18,90 & \\
\hline 3으 $(7,08-23,12)$ & 22,76 & \\
\hline Proporção de indivíduos menores & & $<0,01$ \\
\hline \multicolumn{3}{|l|}{ de 5 anos $(\%)$ ** } \\
\hline $1 ㅇ(0,00-7,72)$ & 15,93 & \\
\hline 2ㅇ $(7,74-9,84)$ & 20,07 & \\
\hline 3으 $(9,87-20,71)$ & 28,18 & \\
\hline Calorias per capita consumidas & & $<0,01$ \\
\hline \multicolumn{3}{|l|}{ no domicílio ** } \\
\hline 1으 $(394,63-1.609,79)$ & 24,14 & \\
\hline 2o $(1.614,19-1.946,93)$ & 21,88 & \\
\hline 3으 $(1.947,64-8.534,94)$ & 17,68 & \\
\hline Total & 21,27 & \\
\hline
\end{tabular}

* Valor de p de tendência linear;

** Variáveis apresentadas em tercis;

*** Apresentada segundo grau de urbanização.

de forma geral como o modelo apresentado na equação 1 adicionada de um termo quadrático da renda), permitindo mudanças não lineares na elasticidade-renda entre os níveis de renda. Nota-se que a elasticidade-renda é particularmente elevada para as menores rendas (cerca de 0,5 no estrato de domicílios com menor renda: $\mathrm{R} \$ 68,70$ per capita/mês), diminuindo progressivamente com o aumento da renda, até ficar próxima a 0,1 no estrato de domicílios com maior renda (R\$ 4.675,10 per capita/mês).

Modelos independentes para as regiões Centro-Sul e Norte-Nordeste foram testados, mas não apresentaram resultados distintos dos encontrados para o total dos domicílios (dados não mostrados). 
Coeficiente de elasticidade-renda e coeficientes de regressão para as variáveis de confusão, relativos à associação entre renda per capita e parcela dos gastos com alimentação fora do domicílio. Brasil, 2002/2003.

\begin{tabular}{lcc}
\hline Variáveis no modelo & Coeficiente de regressão & IC95\% \\
\hline Renda domiciliar per capita & 0,292 & 0,$227 ; 0,356$ \\
Macro-região ( $=$ Norte/Nordeste) & 0,051 & $-0,030 ; 0,131$ \\
Situação do domicílio (0 = Rural) & & 0,$247 ; 0,556$ \\
$\quad$ Capital & 0,401 & 0,$164 ; 0,474$ \\
$\quad$ Região Metropolitana & 0,319 & 0,$144 ; 0,407$ \\
$\quad$ Outras áreas urbanas & 0,275 & 0,$027 ; 0,974$ \\
Proporção do chefe de domicilio do sexo feminino & 0,501 & $-2,135 ; 0,947$ \\
Proporção de indivíduos maiores de 64 anos & $-0,594$ & \\
\hline
\end{tabular}

$\mathrm{R}^{2}=0,628$.

Nota: a proporção de indivíduos menores de cinco anos não atendeu ao critério para inclusão no modelo.

Figura 1

Variação da elasticidade-renda para a parcela dos gastos com alimentação fora do domicílio. Brasil, 2002/2003.

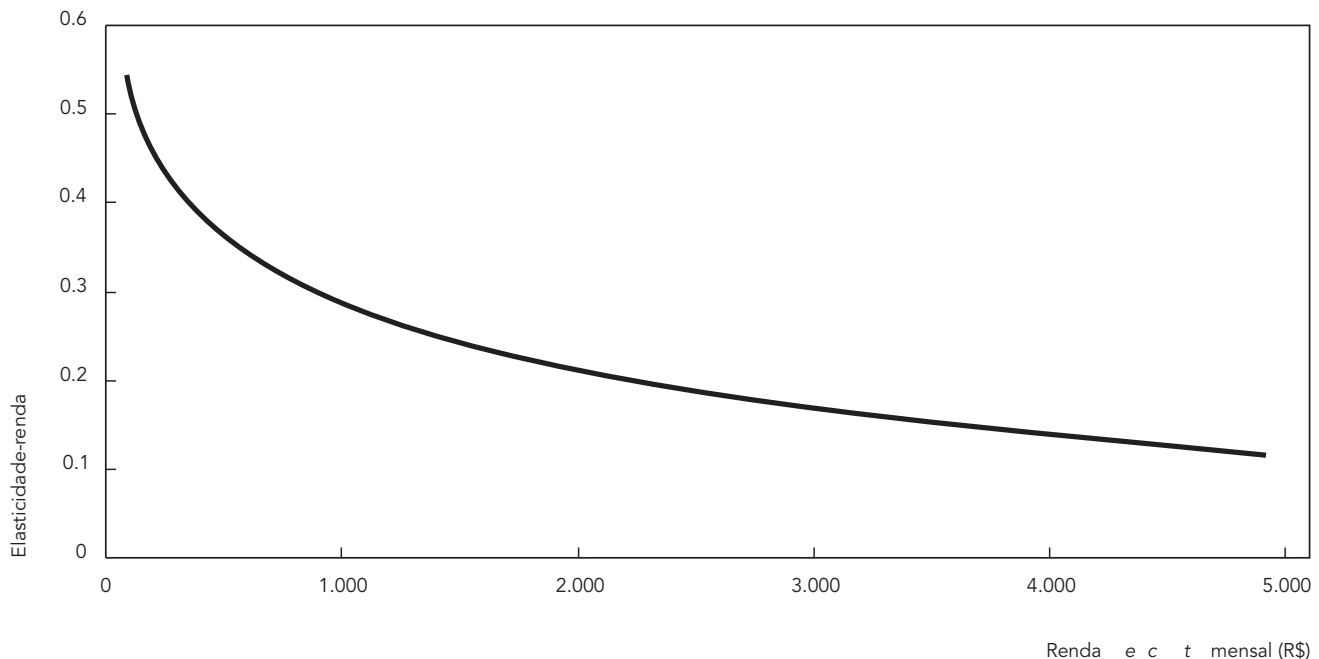

Nota: elasticidade-renda ajustada segundo: situação, macro-região, proporção de indivíduos com mais de 64 anos e proporção de chefe de domicílio do sexo feminino.

\section{Discussão}

Considerando-se o registro das aquisições de alimentos feitos pela POF, observou-se um aumento da parcela da alimentação feita fora do domicílio conforme a elevação da renda familiar, tendo esse efeito permanecido estatisticamente significante após ajuste para características sócio-demográficas e econômicas dos estratos (1\% de aumento na renda aumentaria essa participação, em média, em 0,29\%). Ainda que sempre positivo, o efeito da renda sobre a participação da alimentação fora do domicílio diminui conforme elevação da renda.

A principal limitação do presente estudo se relaciona ao fato de a POF não possibilitar a identificação da quantidade de alimentos verdadeiramente consumida (ou adquirida) fora da mora- 
dia. Por isso, nesta investigação, caracterizou-se a alimentação fora do domicílio por meio de sua participação nos gastos totais com alimentação, e não do consumo alimentar propriamente dito. Ainda assim, não há razões para se crer que o sentido das relações encontradas pudesse ser diferente caso tivesse sido possível avaliar propriamente a aquisição de alimentos para consumo fora do domicílio. Uma vez que a parcela dos gastos com alimentação feita fora de casa se relacionou negativamente com a quantidade de calorias adquirida para consumo no domicílio, é razoável supor que essa variável seja uma boa proxy da quantidade de alimentos consumida fora do lar.

Para fazer face ao curto período de referência da coleta de dados feita em cada residência, este estudo adotou, como unidade de análise, grupos de domicílios homogêneos quanto à localização territorial e atributos sócio-econômicos. A análise das moradias agrupadas em estrato - em vez de isoladas - permitiu que fossem avaliadas unidades com boa amplitude de variação geográfica e sócio-econômica, cujo padrão de aquisição anual de alimentos pôde ser estimado com grande precisão. Com o agrupamento, foi possível, ainda, eliminar grande parte da influência sazonal sobre a aquisição de alimentos, uma vez que os estratos contêm domicílios estudados durante todos os trimestres do ano 12.

Dois pontos fortes desta pesquisa são a representatividade nacional da amostra de domicílios estudada e o controle estatístico de diversos atributos sócio-demográficos, os quais poderiam atuar como fatores de confusão ou de modificação de efeito para a associação entre alimentação fora do domicílio e renda familiar. Ainda assim, com relação a este último aspecto, como em qualquer estudo observacional, não se pode descartar a possibilidade de existência de fatores de confusão não considerados em nossa análise.

Mesmo que pouco explorada na literatura, a alimentação fora do lar apresentou grande importância nas despesas com alimentação das famílias brasileiras (cerca de um quinto). Nos domicílios de maior renda e nas capitais de estados do país, o gasto com alimentação fora de casa já se aproxima de valores apresentados por países desenvolvidos, aproximadamente $30 \%$ do total de despesas com alimentação 15 .

Nosso estudo também possibilita elaborar algumas considerações sobre a relação entre as demais variáveis incluídas em nossas análises e a participação da alimentação fora do domicílio. Esta participação cresceu conforme o nível de urbanização do município e conforme o aumento da proporção de lares chefiados por mulheres no estrato. Nos municípios mais urbanizados, existe uma oferta de restaurantes e serviços de alimentação, facilitando o consumo de alimentos fora do lar 16; além disso, entre os adultos, é comum realizar ao menos uma refeição fora do lar durante o horário de trabalho, em face da dificuldade de deslocamento entre o trabalho e a residência 10 . Do mesmo modo, a proporção de domicílios chefiados por mulheres no estrato pode ser vista como uma aproximação da proporção de mulheres empregadas e responsáveis pelo sustento econômico de seus lares. Nesse caso, a ausência da mulher, geralmente responsável pelo provimento doméstico de alimentação, seria um fator importante para elevação da alimentação fora de casa 17 .

Observa-se, ainda, neste estudo, que o aumento da renda exerce impacto positivo sobre os gastos com alimentação fora do domicílio em todos os níveis de renda. Entretanto, essa influência é máxima entre as residências com menor renda, cujo valor per capita é inferior a $\mathrm{R} \$ 300,00$; neste caso, o aumento de cerca de $10 \%$ na renda poderia aumentar a participação dos gastos totais com alimentação fora do domicílio, em média, em mais de $4,5 \%$. Ainda que esta pesquisa não avalie a evolução da parcela dos gastos com alimentação feita fora do domicílio no país, nas últimas décadas, acredita-se que esta tenha aumentado seguindo a melhora do poder aquisitivo vivenciado no Brasil nesse período. Desse modo, os gastos com alimentação fora do domicílio podem, inclusive, auxiliar na explicação da evolução desfavorável no padrão de aquisição domiciliar de alimentos nas áreas metropolitanas do Brasil 2 .

Inúmeros estudos evidenciaram que a alimentação fora de casa tem maior densidade energética, com maiores quantidades de gorduras, gorduras saturadas e menor quantidade de micronutrientes 6,18,19. Além disso, a maior participação da alimentação fora do domicílio também se associa a maiores prevalências de excesso de peso 18,20,21 e sedentarismo 15 .

Embora este artigo tenha se dedicado a estudar especificamente o papel da renda na determinação da alimentação fora do lar, outros aspectos observados no Brasil também podem influenciar o padrão de gastos com alimentação. Entre eles, a redução no preço da alimentação fora de casa, o acesso a mais estabelecimentos de venda de alimentos e a expansão das redes de fast-food 22,23, os quais, junto à melhora da renda da população, atuam de forma a torná-la cada vez mais acessível a segmentos de menor renda da população.

Por fim, os resultados deste estudo apontam para uma importante influência da renda na parcela dos gastos com alimentação realizada fora 
do domicílio, sugerindo que a evolução favorável da renda, principalmente nos estratos mais pobres da população, resultará em aumento da participação dessa forma de se alimentar, acarretando, possivelmente, redução da qualidade nutricional da alimentação no país.

\section{Resumo}

Objetivou-se descrever e avaliar a influência da renda sobre a participação da alimentação fora do domicílio no Brasil. Utilizaram-se dados coletados pela Pesquisa de Orçamentos Familiares realizada em 2002/2003 (POF 2002/2003), pelo Instituto Brasileiro de Geografia e Estatística. Analisaram-se os registros dos gastos com aquisições de alimentos e bebidas consumidos fora do domicílio. A associação entre a participação da alimentação fora do domicílio e a renda, ajustada para atributos sócio-demográficos, foi estudada por meio de modelos de regressão utilizados para estimação de coeficientes de elasticidade-renda. A alimentação fora do domicílio representou $21 \%$ do total dos gastos com alimentação; destaque-se que o incremento de $10 \%$ na renda aumentaria em 3\% a participação da alimentação fora do domicílio. O efeito da renda sobre a participação da alimentação fora, ainda que sempre positivo, diminui conforme elevação da renda, sendo alto nos domicílios com renda inferior a $R \$ 68,70$ per capita/mês. Há influência da renda nos gastos com alimentação fora do domicílio, assim a evolução favorável da renda resultará em aumento dessa forma de se alimentar.

Renda; Inquéritos Nutricionais; Consumo de Alimentos; Alimentação

\section{Colaboradores}

R. M. Claro, R. B. Levy e D. H. Bandoni contribuíram para o planejamento do estudo, na análise dos dados e na redação do artigo. 


\section{Referências}

1. Mondini L, Monteiro CA. Mudanças no padrão de alimentação da população brasileira (1962-1988). Rev Saúde Pública 1994; 28:433-9.

2. Levy-Costa RB, Sichieri R, Pontes NS, Monteiro CA. Disponibilidade de alimentos no Brasil: distribuição e evolução (1974-2003). Rev Saúde Pública 2005; 39:530-40.

3. World Health Organization. Diet, nutrition and the prevention of chronic diseases. Geneva: World Health Organization; 2003. (WHO Technical Report Series, 916).

4. Bleil SI. O padrão alimentar ocidental: considerações sobre a mudança de hábitos no Brasil. Cadernos de Debate 1998; VI:1-25.

5. French SA, Story M, Jeffery RW. Environmental influences on eating and physical activity. Annu Rev Public Health 2001, 22:309-35.

6. Lin B, Frazão E, Guthrie J. Away-from-home foods increasingly important to quality of American diet. Washington DC: Food and Rural Economics Division, Economic Research Service; 1999. (U.S. Department of Agriculture Information Bulletin, 749).

7. Harnack LJ, Jeffery RW, Boutelle KN. Temporal trends in energy intake in the United States: an ecologic perspective. Am J Clin Nutr 2000; 71:1478-84.

8. Guthrie JF, Lin BH, Frazão E. Role of food prepared away from home in the american diet, 1977-78 versus 1994-96: changes and consequences. J Nutr Educ Behav 2002; 34:140-50.

9. Ma H, Huang J, Fuller F, Rozelle S. Getting rich and eating out: consumption of food away from home in urban China. Canadian Journal of Agricultural Economics 2006; 54:101-19.

10. Paulin GD. Let's do lunch: expenditures on meals away from home. Mon Labor Rev 2000, 5:36-45.

11. Monteiro CA, Mondini L, Levy-Costa RB. Mudanças na composição e adequação nutricional da dieta familiar nas áreas metropolitanas do Brasil (1988-1996). Rev Saúde Pública 2000; 34:251-8.

12. Instituto Brasileiro de Geografia e Estatística. Pesquisa de Orçamentos Familiares 2002-2003: análise da disponibilidade domiciliar de alimentos e do estado nutricional no Brasil. Rio de Janeiro: Instituto Brasileiro de Geografia e Estatística; 2004.

13. Guo X, Popkin BM, Mroz TA, Zhai F. Food price can favorably alter macronutrient intake in China. J Nutr 1999; 129:994-1001.
14. Claro RM, Carmo HCE, Machado FMS, Monteiro CA. Renda, preço dos alimentos e participação de frutas e hortaliças na dieta. Rev Saúde Pública 2007; 41:557-64.

15. Orfanos PH, Naska A, Trichopoulos D, Slimani N, Ferrari P, van Bakel M. Eating out of home and its correlates in 10 European countries, the European Prospective Investigation into Cancer and Nutrition (EPIC) study. Public Health Nutr 2007; 10:1515-25.

16. Beydoun MA, Powell LM, Wang Y. Reduced awayfrom-home food expenditure and better nutrition knowledge and belief can improve quality of dietary intake among US adults. Public Health Nutr 2008; 12:369-81.

17. Nayga RM. Wife's labor force participation and family expenditures for prepared food, food prepared at home, and food away from home. Agricultural and Resource Economics Review 1996; 25:179-86.

18. Kant AK, Graubard BI. Eating out in America, 19872000: trends and nutritional correlates. Prev Med 2004; 38:243-9.

19. Kearney JM, Hulshof KFAM, Gibney MJ. Eating patterns temporal distribution, converging and diverging foods, meals eaten inside and outside of the home - implications for developing FBDG. Public Health Nutr 2001; 4:693-8.

20. Nielsen S, Popkin B. Changes in beverage intake between 1977 and 2001. Am J Prev Med 2004; 27:205-10.

21. Ma Y, Bertone ER, Stanek EJ, Reed GW, Hebert JR, Cohen NL, et al. Association between eating patterns and obesity in a free-living US adult population. Am J Epidemiol 2003; 158:85-92.

22. Garcia RWD. Reflexos da globalização na cultura alimentar: considerações sobre as mudanças na alimentação urbana. Rev Nutr 2003; 16:483-92.

23. Bowman SA, Vinyard BT. Fast food consumption of U.S. adults: impact on energy and nutrient intakes and overweight status. J Am Coll Nutr 2004; 23:63-8.

Recebido em 23/Abr/2009

Versão final reapresentada em 28/Jul/2009

Aprovado em 19/Ago/2009 